\title{
Identification of miRNAs in a Model of Retinal Degenerations
}

\author{
Kartik Saxena, Matthew Viktor Rutar, Jan M. Provis, and Riccardo Carlo Natoli \\ The Australian National University, Canberra, Australia
}

Correspondence: Riccardo Carlo Natoli, College of Medicine, Biology and Environment, Medical School and The John Curtin School of Medical Research (Building 131), Department of Neuroscience, The Australian National University, Canberra, ACT 0200, Australia; riccardo.natoli@anu.edu.au.

Submitted: August 14, 2014

Accepted: February 13, 2015

Citation: Saxena K, Rutar MV, Provis JM, Natoli RC. Identification of miRNAs in a model of retinal degenerations. Invest Ophthalmol Vis Sci. 2015;56:1820-1829. DOI:10.1167/ iovs.14-15449
PuRPose. We investigated the expression profile of and identify all microRNAs (miRNAs) that potentially regulate inflammation in a light-induced model of focal retinal degeneration.

Methods. Sprague Dawley (SD) rats aged 90 to 140 postnatal days were exposed to 1000 lux white fluorescent light for 24 hours. At 24 hours, and 3 and 7 days after exposure, the animals were euthanized and retinas processed for RNA. Expression of 750 miRNAs at 24 hours of exposure was assessed using low density array analysis. Significantly modulated miRNAs and their target mRNAs were used to assess the potential biological effects. Expression of seven miRNAs, potentially modulating inflammation, was investigated across a protracted time course after light exposure using quantitative PCR. Photoreceptor cell death was analyzed using TUNEL.

Results. Intense light exposure for 24 hours led to differential expression of a number of miRNAs, 37 of which were significantly modulated by 2 -fold or more. Of those, 19 may potentially regulate the inflammatory immune response observed in the model. MicroRNAs $-125-3 p,-155,-207,-347,-449 a,-351$, and $-542-3 p$ are all upregulated at 24 hours of exposure along with peak photoreceptor cell death. The MiRNAs $-542-3 \mathrm{p}$ and -351 reached maximum expression at 7 days after exposure, while $-125-3 p,-155,-207,-347$, and -449 reached a peak expression at 3 days.

Conclusions. The results of the study show that miRNAs are modulated in response to light damage (LD). These miRNAs potentially regulate the inflammatory immune response, triggered as a result of the acute retinal damage, which is a key mediator of retinal degeneration in this model and age-related macular degeneration.

Keywords: AMD, microRNA, light damage, retinal degeneration, noncoding RNA
M icroRNAs (miRNAs) are small evolutionarily conserved noncoding RNA sequences that modulate a range of biological processes, ${ }^{1}$ including cell death ${ }^{2,3}$ and inflammation. ${ }^{4}$ They are approximately 22 to 25 nucleotides long and provide a second layer of posttranscriptional gene regulation by targeting messenger RNAs (mRNAs) for degradation or repression of translation, ${ }^{5}$ and are found in abundance in the central nervous system (CNS). ${ }^{6,7}$

Age-related macular degeneration (AMD) is a progressive degenerative disease of the retina that causes irreversible vision loss and accounts for up to $50 \%$ of central blindness cases worldwide. The involvement of inflammatory processes in the pathogenesis of AMD was documented in the $1980 \mathrm{~s}^{8}$ and its central role confirmed by gene association studies. ${ }^{9-11}$ Subsequently, a large number of inflammatory factors and cofactors, particularly in the complement pathways, have been implicated in the disease process (see review ${ }^{12}$ ).

The acute retinal light damage (LD) model in rats has features in common with dry AMD,${ }^{13,14}$ including cell death and inflammation. ${ }^{14}$ This model has been used to investigate the involvement of chemokines and macrophages in the progression of retinal degeneration. ${ }^{15}$ Following LD, a lesion formed by the death of photoreceptors and atrophy of the RPE presents on the visual axis and enlarges over time, even in the absence of the damaging stimulus. ${ }^{14}$ In this model photoreceptor death is associated with expression of chemokines by
Müller glia, recruitment of macrophages, and deposition of $\mathrm{C} 3,{ }^{16}$ and attenuation of macrophage recruitment, by silencing expression of the chemokine $\mathrm{Ccl} 2$, reduces photoreceptor cell death. ${ }^{17}$ Similar patterns of chemokine expression, macrophage recruitment, C3 deposition, and photoreceptor cell death are present in the normally aging rat, ${ }^{18}$ indicating that the model represents many of the features of normal aging of the retina.

Recent evidence supports the idea that miRNAs are involved in inflammation. ${ }^{19,20}$ Many miRNAs are rapidly upregulated in response to inflammatory cues and may either promote the duration and magnitude of inflammation ${ }^{21}$ or silence it. ${ }^{22}$ In this study we investigated the modulation of miRNAs in the rat LD model of focal retinal degeneration and explored the transcriptional profile of their target genes in the retina following LD, with a focus on identifying miRNAs that modulate expression of genes involved in the inflammatory response.

\section{MeTHODS}

\section{Animals and Light Damage}

Animal handling and treatment protocols were done in accordance with the ARVO Statement for the Use of Animals in Ophthalmic and Vision Research, and were approved by the Australian National University (ANU) Animal Ethics Committee. Sprague Dawley (SD) rats aged 90 to 140 postnatal days raised 
in $\operatorname{dim}$ (5 lux) cyclic light ("dim-reared") were used for the study. Light damage was induced by exposure to bright light (1000 lux) from an overhead white fluorescent source (COLDF2 $2 \times 36 \mathrm{~W}$ IHF; Thorn Lighting, Brisbane, Australia) for a period of 24 hours. Food was provided ad libitum. At the end of the exposure period animals were either euthanized immediately by intraperitoneal injection of barbiturate overdose (60 mg/kg bodyweight, Valabarb; Virbac, Carros, France), or were returned to dim light ( $5 \mathrm{lux}$ ) conditions for 3 or 7 days before euthanasia. Retinal tissue was collected immediately for analysis. Age-matched, dim-reared animals were used as controls. All experimental groups were $n=5$, unless otherwise stated.

\section{Tissue Collection}

The retina from the right eye of each animal was excised through a corneal incision and placed in RNAlater solution (Ambion Biosystems, Austin, TX, USA), stored at $4^{\circ} \mathrm{C}$ overnight then transferred to $-80^{\circ} \mathrm{C}$. The left eye from each animal was enucleated, the superior margin marked, then immersed in $4 \%$ paraformaldehyde for 3 hours at $4{ }^{\circ} \mathrm{C}$. The anterior segment was removed, then the eye cups replaced in fresh $4 \%$ paraformaldehyde overnight at $4^{\circ} \mathrm{C}$, and subsequently prepared for paraffin embedding. Eyes were sectioned at $6 \mu \mathrm{m}$ on a microtome on the vertical axis. Only sections containing the optic nerve were used for analysis.

\section{RNA Extracted From Rat Retina}

Total RNA was extracted from retinal samples using the mirVana miRNA isolation kit (Ambion Biosystems), according to manufacturer's protocol. The concentration of the RNA was determined by ND-1000 spectrophotometer (Nanodrop Technologies, Wilmington, DE, USA) and quality using the 2100Bioanalyser (Agilent Technologies, Santa Clara, CA, USA). Only RNA samples with a A260/A280 ratio above 1.9 and an RNA integrity number (RIN) greater than 8.0 were used for the study. The RNA samples were stored at $-80^{\circ} \mathrm{C}$ before performing TaqMan miRNA array studies.

\section{TaqMan miRNA Array and Analysis}

The RNA from dim-reared control retinas and 24-hour lightexposed retinas were used for miRNA array card analysis. Total RNA ( $700 \mathrm{ng}$ ) from each animal was reverse transcribed to two different cDNA pools (each containing $350 \mathrm{ng}$ of miRNAspecific cDNA) using the Megaplex RT Primers, Rodent Pool A and B Set v3.0 (Applied Biosystems, Carlsbad, CA, USA) and TaqMan miRNA RT kit (Applied Biosystems) according to manufacturer's protocol; a $7.5-\mu \mathrm{L}$ reaction mixture, including $50 \mathrm{U}$ Multiscribe Reverse Transcriptase and $20 \mathrm{U}$ RNase inhibitor. Card A contains well characterized miRNAs in miRBase v16.0 (available in the public domain at www. mirbase.org), while B contains uncharacterized ones. Both A and $B$ samples then were hybridized to the respective TaqMan Array Rodent MicroRNA Cards v3.0 (A and B), which are preconfigured microfluidic 384-well format plates. Each well in the microfluidic card consists of a TaqMan chemistry-based primer probe set for a unique miRNA or other RNA (control sequences). A total of 20 microRNA cards was run (10 A cards and $10 \mathrm{~B}$ cards). The microfluidic cards then were run on the ViiA 7 Real-time PCR machine (Applied Biosystems) to generate the raw expression data.

The expression data were compiled and analyzed using PARTEK Genomic Suite 6.6 software (Partek, Inc., St. Louis, MO, USA). Amplification data for the target miRNAs were first normalized by subtracting the endogenous control (Y1) values.
TABLE 1. TaqMan Small RNA Probes Used

\begin{tabular}{llc}
\hline \multicolumn{1}{c}{ miRNA ID } & \multicolumn{1}{c}{ Accession \# } & Product \# \\
\hline mmu-miR-155 & MIMAT0000165 & 002571 \\
hsa-miR-125b-3p & MIMAT0004592 & 002378 \\
mmu-miR-351 & MIMAT0000609 & 001067 \\
mmu-miR-542-3p & MIMAT0003172 & 001284 \\
hsa-miR-449a & MIMAT0001541 & 001030 \\
rno-miR-347 & MIMAT0000598 & 001334 \\
mmu-miR-207 & MIMAT0000240 & 001198 \\
U6 snRNA & NR_004394 & 001973 \\
mmu-miR-182 & MIMAT0005300 & 002599 \\
hsa-miR-183 & MIMATO000860 & 002269 \\
mmu-miR-96 & MIMAT0000818 & 000186 \\
mmu-miR-467d & MIMAT0004886 & 002518 \\
\hline
\end{tabular}

Differential expression was examined using the 1-way ANOVA statistic with a significance cutoff of $P<0.05$. The statistical robustness of the expression data was visualized with principle component analysis (PCA), provided within the Partek Genomic Suite 6.6 software. MicroRNA expression distribution was visualized using the volcano plot tool embedded within the Partek software. A list of significantly regulated miRNAs was generated from the volcano plot by selecting for miRNAs that changed $\geq 2$-fold and had a $P$ value of $<0.05$. These highly modulated miRNAs were used for biological functional analyses using PARTEK Genomic Suite 6.6.

\section{Biologic Functional Analyses}

We used data from a previous microarray analysis, ${ }^{23}$ identifying genes modulated by light damage in this same LD model, to monitor up- or downregulation of the predicted target genes of the miRNAs found to be significantly regulated in the present study. The pre-existing microarray data were imported into Partek along with the Affymetrix CEL files to compare the two expression data. The predicted targets were determined using TargetScan v6.2 embedded in the PARTEK Genomic Suite 6.6 software (Partek, Inc.). Then, the data were subjected to functional analysis via Gene Ontology (GO) enrichment provided in PARTEK Genomic Suite 6.6 and clustered according to biological processes.

\section{Quantitative Real-Time PCR}

The cDNA was synthesized using the TaqMan MicroRNA RT kit (Applied Biosystems) according to manufacturer's protocol; a $15-\mu \mathrm{L}$ reaction mixture, including $500 \mathrm{ng}$ to $1 \mu \mathrm{g} \mathrm{RNA}, 50 \mathrm{U}$ Multiscribe Reverse transcriptase, $3 \mu \mathrm{L} 5 \times$ miRNA specific RT primer and $3.8 \mathrm{U}$ RNase Inhibitor. The miRNA amplification was measured using commercially available miRNA specific TaqMan hydrolysis probes (Applied Biosystems) detailed in Table 1 . The hydrolysis probes were used according to the manufacturer's directions in a $10-\mu \mathrm{L}$ reaction mix along with TaqMan Gene Expression Mastermix and the cDNA. Fluorescence was measured by the FAM $510 \mathrm{~nm}$ channel in the 7900 HT Real-time PCR machine (Applied Biosystems), ROX passive reference dye present in the Gene Expression Mastermix was used to normalize samples in individual wells. Each biological sample was amplified in a technical replicate and the average critical threshold cycle $\left(\mathrm{C}_{t}\right)$ value was used to determine the change in expression. Fold change was calculated using the $\Delta \Delta \mathrm{C}_{\mathrm{t}}$ method, where target miRNAs were normalized to the expression of small nuclear RNA U6 (reference RNA), which showed no differential expression in this study. Gel electrophoresis was used to access amplification specificity, and statistical analysis was performed using 1-way ANOVA and 

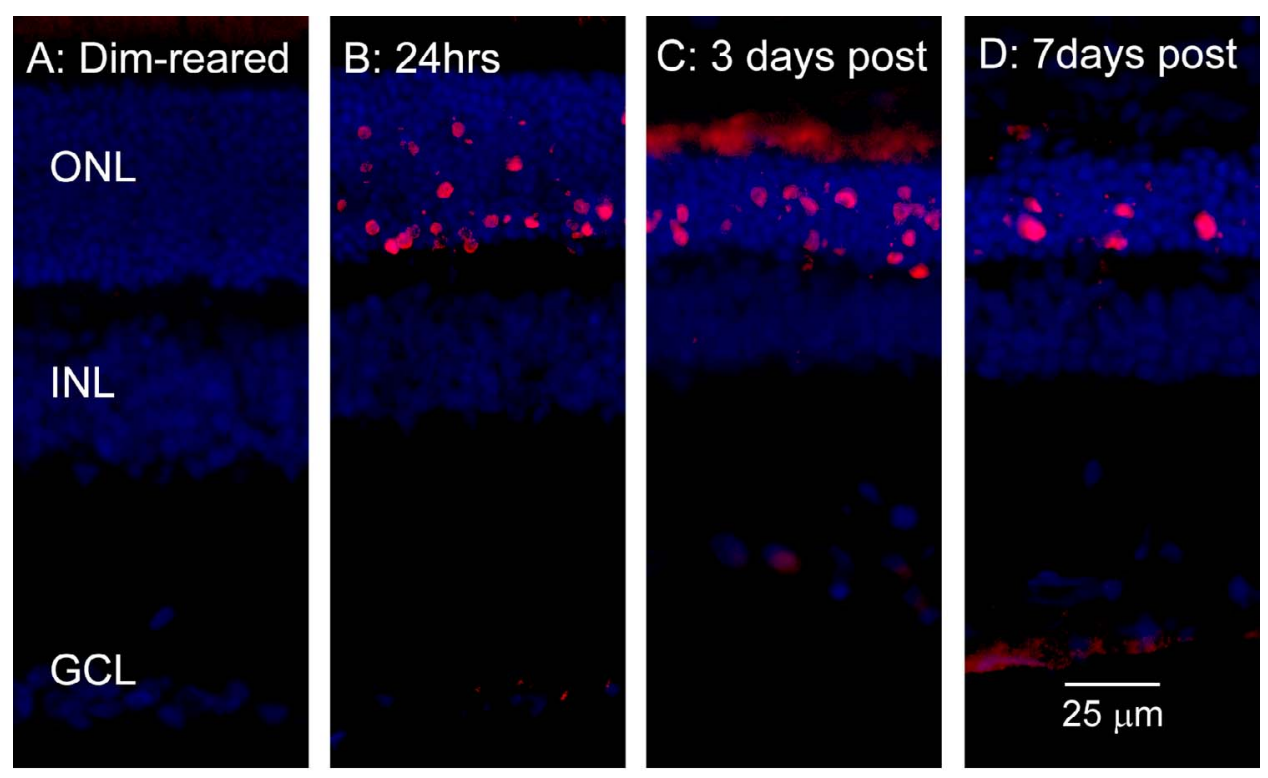

\section{E: TUNEL Positive Cell Counts}

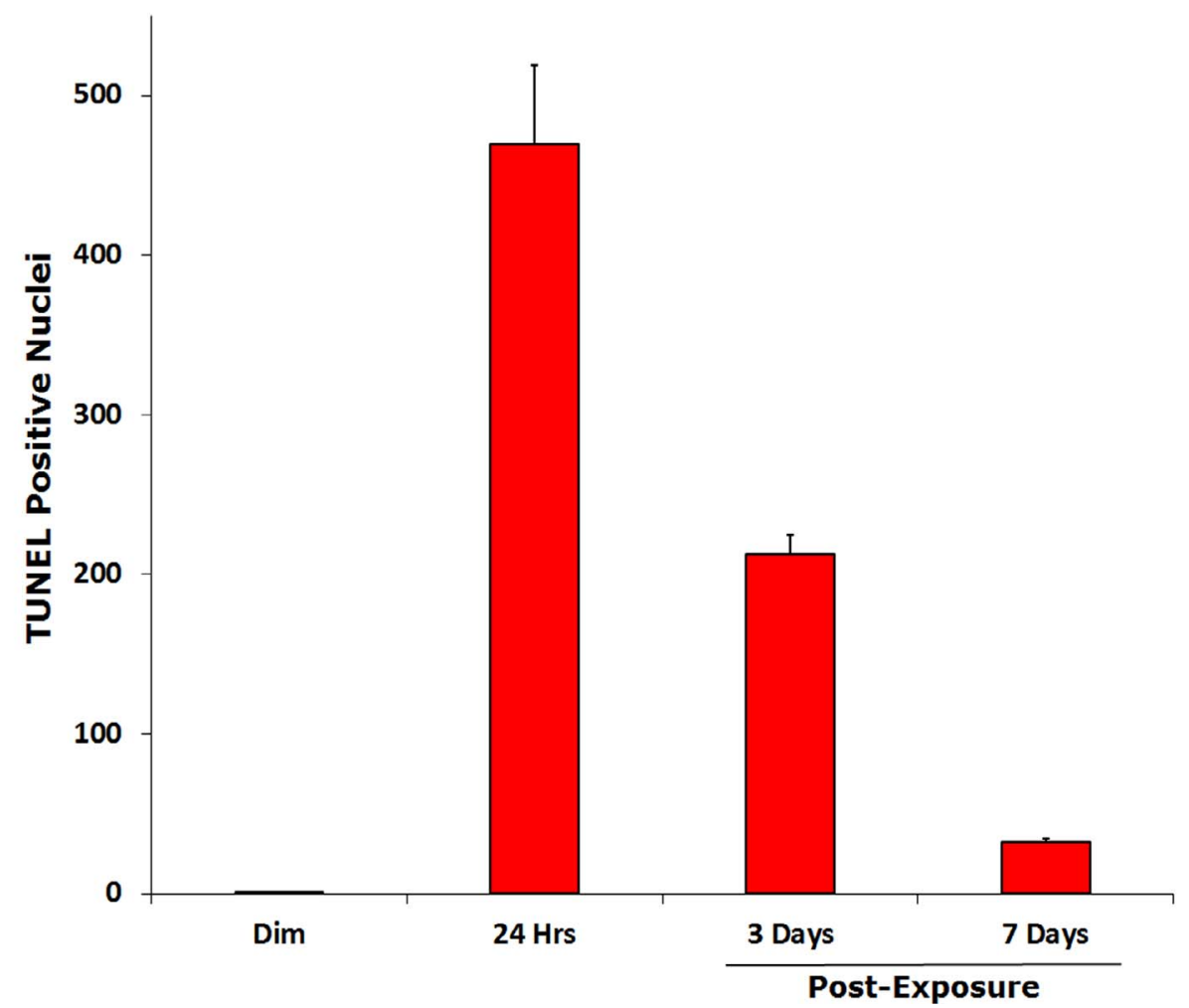

FigURE 1. The number of TUNEL-positive photoreceptor nuclei in the ONL was used to estimate progression of retinal degeneration at three time points. Representative TUNEL staining images are documented in (A-D), while total number of TUNEL-positive cells (across the entire retinas) is quantified in (E).

Student's $t$-test, using Prism (GraphPad Software V5; GraphPad Software, Inc., La Jolla, CA, USA).

\section{Analysis of Cell Death}

We used the TUNEL labeling techniqueto quantify cell death over the LD time course, in retinal cryosections using a previously published protocol. ${ }^{23}$ The TUNEL-positive cells in the outer nuclear layer (ONL) were counted across the full length of the retinal sections cut in the vertical meridian, including at the optic nerve head. Cells were counted at 1-mm intervals across retinal sections, the final count from each animal was averaged from at least two sections, with four or five animals analyzed for each experimental condition. Statistical analysis was performed using 1-way ANOVA and Student's $t$-test, using Prism (GraphPad Software V5). 


\section{Principle Component Analysis PCA Separation score - $50.3 \%$}

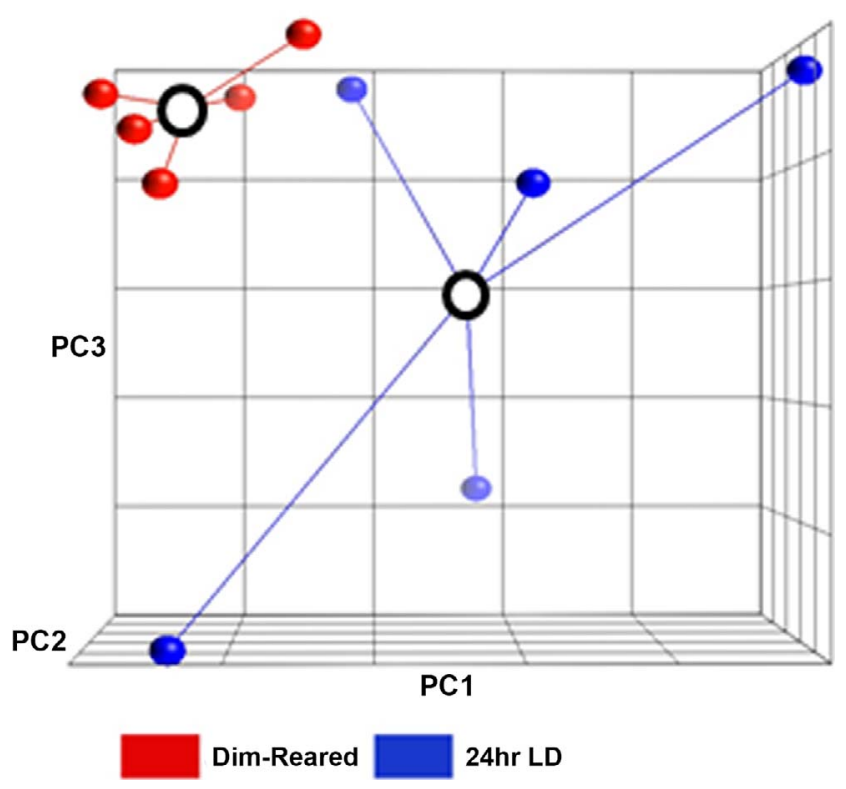

Figure 2. The 3-D principle component analysis. Each sphere represents a sample and the circle represents the centroid of each distribution. Samples from animals not exposed to LD (red) are more tightly clustered than samples from LD animals (blue), indicating less variability in miRNA expression profiles in control animals compared to animals exposed to LD. The majority of the variance between the two different conditions is explained by the PC-1 axis.

\section{Results}

\section{TUNEL Analysis}

There was an evident increase in the number of photoreceptor cells undergoing apoptosis following exposure to 24 hours of bright light, consistent with our previous reports (Fig. 1). This increase was more prominent in the superior retina approximately $2 \mathrm{~mm}$ superotemporal to the optic disc, shown in the representative image panels (Figs. 1A-D). We observed a peak in the number of TUNEL+ cells in the ONL after 24 hours of bright light exposure followed by a significant decrease during the postexposure periods, 3 and 7 days, as well as a progressive thinning of the ONL/photoreceptor layer as described previously. ${ }^{15}$

\section{MicroRNA Profiling}

The PCA plot (Fig. 2) shows that the biological replicates are clustered close together and that the majority of variation between the Control and LD groups (50.3\%) was due to LD, rather than inherent variability within the groups. The volcano plot (Fig. 3) shows that a large number of miRNAs were modulated up or down by LD. A list of candidate miRNAs (Table 2) was compiled by selecting those with a change of $\geq 2$ or $\leq-2$-fold, and $P<0.05$. A total of 37 miRNAs of the 750 tested showed strong statistical significance and were selected for further analysis.

The most highly upregulated miRNA was mmu-miR-467d (66-fold), while mmu-miR-1224_mat was the most downregulated (-16-fold). Of the 37 miRNAs, 26 were upregulated and 11 downregulated; 17 came from the miRNA array card A, which represents the better characterized miRNAs in miRBase v16.0 (available in the public domain at www.mirbase.org), while 20 were from the not-so-well characterized group on array card $\mathrm{B}$.

\section{Functional Analysis of miRNAs}

Each miRNA targets multiple mRNAs; therefore, the list of predicted gene targets is much larger than the list of significantly regulated miRNAs. The list of gene targets (from the 2300 genes analyzed) of the 37 miRNAs were analyzed using PARTEK, to gain insight into their biological relevance by GO enrichment clustering based on "biological processes." Through this process we identified the 10 most highly represented gene ontology clusters (Table 3). The "biological processes" with the highest enrichment scores include positive regulation of cell proliferation (22.5502), inflammatory response (19.9534), positive regulation of transcription from RNA polymerase II promoter (19.7899), and angiogenesis (19.3955). Table 4 shows the 19 miRNAs associated with "inflammatory response" and identifies the 30 gene targets of those miRNAs. Functional clustering of those genes using the DAVID Bioinformatics annotation tool 6.7 identifies 7 of 30 genes clustering into two families of chemokines; the $\mathrm{Ccl}(3,4$, 7 , and 12) and $\mathrm{Cxcl}(1,10$, and 11) families.

\section{Temporal Expression of Inflammation-Associated miRNAs}

Based on the expression signal-to-noise ratio (Critical $F$ value $=$ 2.55), consistency across biological replicates, we selected eight miRNAs involved in regulating inflammatory responses for validation by quantitative PCR (qPCR). We tested the expression levels of miR-125-3p, miR-155, miR-207, miR-351, miR-449a, miR-542-3p, miR-467d, and rno-miR-347 at three time points: 24 hours of light exposure, and 3 and 7 days after exposure (Figs. 4A-C). In addition to these, we verified the expression pattern of mRNA cluster miR-183/96/182 at the three time points (Fig. 4D), which has been shown previously to express selectively in photoreceptors, is modulated in rodent models of retinal degenerations, and due to environmental light conditions.

Two different expression patterns of the miRNA subset were detected across the time points. Five miRNAs (miR-207, $-347,-125 b-3 p,-155$, and $-449 a)$ reached peak expression at 3 days after exposure (Figs. 4A, 4B); in contrast, two miRNAs, (miR-542-3p and -351) were continuously upregulated over the time course (Fig. 4C). We were unable to generate consistent fold change values for miR-467d across the biological replicates due to very high (end stage) $\mathrm{C}_{\mathrm{t}}$ cycle numbers.

MicroRNA cluster miR-183/96/182 exhibited a downward expression trend across the LD time points, with all three miRNAs showing significant reduction in expression at the 3day time point. Only miR-183 and -96 expression reduced significantly at the 24-hour mark, while only miR-183 and -182 showed significant reduction at 7 days after LD.

\section{Discussion}

The results of this study showed that miRNAs are modulated in response to LD. Indeed, two of the most highly regulated gene clusters targeted by these miRNAs are "positive regulation of the transcription from RNA polymerase II promoter" and "negative regulation of DNA dependent transcription." This essentially reflects that light damage causes regulation of the retinal transcriptome. The other clusters include genes that regulate cell proliferation, cell adhesion, angiogenesis, and our target category, the inflammatory response. 


\section{Volcano Plot (LD vs Control)}

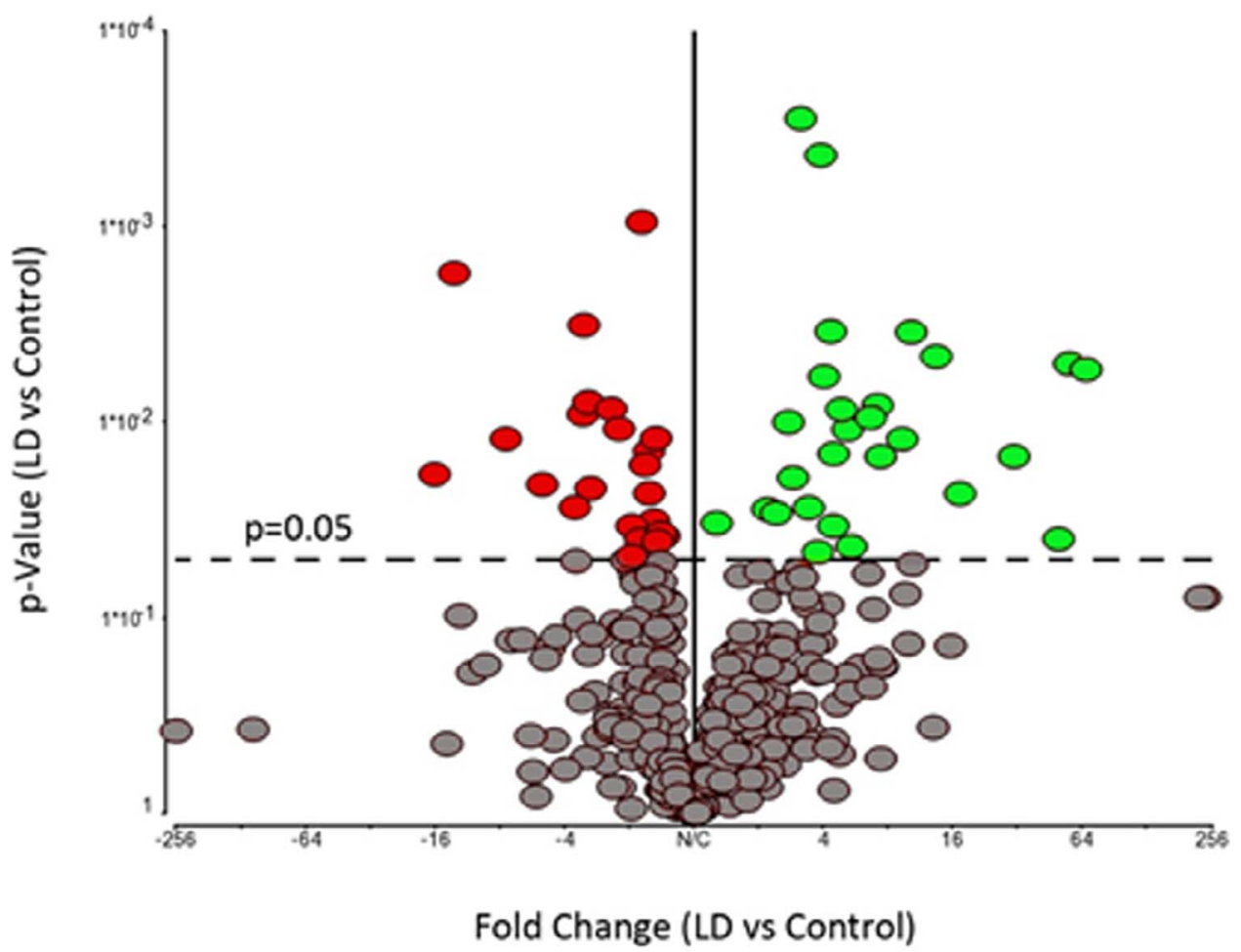

Significantly up-regulated miRNAs

Significantly down-regulated miRNAs

Figure 3. Volcano plot of miRNA expression levels against probability. Only data points lying above the dotted line are significant ( $P \leq 0.05)$. The solid vertical line indicates zero change in gene expression. Points to the right (green) represent candidates that were upregulated by LD, while points to the left (red) were downregulated.

\section{Features of the Light Damaged Retina}

The LD-mediated retinal degeneration has been studied extensively since the landmark study of Noell et al. ${ }^{24}$ in 1966. In LD, photoreceptors degenerate in the superotemporal portion of the rat retina, at the area centralis, where there is a peak density of cones, and ganglion cell density is at its highest. ${ }^{25,26}$ In this respect, the area centralis is homologous to the human macula. Furthermore, the protracted degeneration of photoreceptors and RPE cells triggered by LD in rat retina, and the associated breakdown of the blood retina barrier (BRB) mimic certain histopathological features of dry AMD. ${ }^{13,14,27}$ Oxidative damage and inflammation have roles in the pathophysiology of light-induced retinal degeneration.

Cell Proliferation and Angiogenesis in LD. A surprising finding of the functional clustering analysis is the high representation of target genes involved in cell proliferation and angiogenesis, since neither of these functions feature prominently in the LD model. There are two possible explanations for this. The first is that, because a single miRNA can regulate translation of multiple genes, the list of "target genes" introduced into Partek for clustering is not necessarily the actual targets of the miRNAs we have identified. For example, miR-449a is enriched in "inflammatory response" and "angiogenesis" clusters exhibiting putative binding sites for interleukin 23 receptor mRNA, involved in the immune response, and angiopoietin- 1 receptor mRNA, which facilitates blood vessel formation.

A second possibility is that genes in cell proliferation pathways and/or angiogenic pathways may be upregulated so that the tissues are induced into a "pro-angiogenesis" or "proproliferation" state, without activating all the genes required for angiogenesis or proliferation to take place. Proliferation of non-neuronal retinal cells (astrocytes, microglia, and Müller cells) has been well documented in retinal degenerations, including AMD as a response to intense retinal stress. ${ }^{28-30}$ While angiogenesis has not been reported in the LD model previously, it is possible that leukostasis could cause local hypoxia leading to modulation of angiogenic factors, the effects of which might only be detected following much longer survival periods than explored here.

Cell Adhesion and Inflammation in LD. Cell adhesion has a role in a variety of different biological functions, including cell-cell interactions that are part of the immunological response. It now is well established that retinal degeneration that follows LD in this model is mediated by significant inflammatory processes, and the high representation of gene targets in this functional cluster might be expected on this basis. We have previously identified a range of inflammatory genes upregulated following LD. These include several that encode proteins of the complement system (CFD, C3, C1s, C4b, C5r1) along with several chemokines/cytokines (Ccl2, MCP-3, A21a, A6) ${ }^{16,17,31-33}$ involved in recruitment of 
TABle 2. Candidate miRNAs

\begin{tabular}{|c|c|c|c|}
\hline miRNA ID & $P$ Value & Fold Change & F Statistic \\
\hline mmu-miR-467d & 0.00537844 & 66.934 & 9.22497 \\
\hline mmu-miR-155 & 0.00502399 & 55.9242 & 6.02627 \\
\hline mmu-miR-466h & 0.0395309 & 49.8464 & 2.27494 \\
\hline mmu-miR-220 & 0.0149566 & 30.91 & 3.98379 \\
\hline mmu-miR-125b-3p & 0.0231669 & 17.3396 & 2.54936 \\
\hline mmu-miR-207 & 0.00461902 & 13.4394 & 5.0048 \\
\hline mmu-miR-449a & 0.00346565 & 10.2821 & 4.96975 \\
\hline mmu-miR-467c & 0.0121979 & 9.33694 & 3.5032 \\
\hline mmu-miR-291-5p & 0.0149867 & 7.41014 & 2.6742 \\
\hline mmu-miR-542-3p & 0.00820295 & 7.16889 & 4.76784 \\
\hline mmu-miR-715 & 0.00948857 & 6.65874 & 3.27612 \\
\hline rno-miR-347 & 0.0431166 & 5.4159 & 2.71173 \\
\hline mmu-miR-494 & 0.0108949 & 5.20176 & 3.94319 \\
\hline mmu-miR-351 & 0.00859523 & 4.86756 & 3.45553 \\
\hline mmu-miR-1894-3p_mat & 0.0145028 & 4.48047 & 3.77757 \\
\hline mmu-miR-582-3p & 0.0339127 & 4.4609 & 8.80396 \\
\hline hsa-miR-223 & 0.00343764 & 4.34528 & 6.58455 \\
\hline mmu-miR-685 & 0.00584433 & 4.03733 & 6.29025 \\
\hline mmu-miR-1971_mat & 0.000432933 & 3.9048 & 8.13198 \\
\hline mmu-miR-300 & 0.0460522 & 3.78183 & 3.94242 \\
\hline mmu-miR-1195 & 0.0273212 & 3.43057 & 2.88671 \\
\hline mmu-miR-2183_mat & 0.000281241 & 3.15076 & 13.0543 \\
\hline mmu-miR-509-3p & 0.0192239 & 2.9015 & 2.50254 \\
\hline mmu-miR-335-3p & 0.00999525 & 2.76217 & 5.50385 \\
\hline hsa-miR-214 & 0.0291568 & 2.42738 & 2.23578 \\
\hline mmu-miR-466k_mat & 0.0278496 & 2.2071 & 3.31638 \\
\hline mmu-miR-720 & 0.0108249 & -2.22245 & 3.07113 \\
\hline mmu-miR-376b\# & 0.00855768 & -2.41404 & 3.26994 \\
\hline hsa-miR-124\# & 0.0217727 & -3.01279 & 2.69783 \\
\hline hsa-miR-411\# & 0.00789667 & -3.08945 & 3.39888 \\
\hline mmu-miR-337 & 0.00319273 & -3.23811 & 4.88565 \\
\hline mmu-miR-466d-5p & 0.00911546 & -3.27501 & 4.25185 \\
\hline rno-miR-743a & 0.0272182 & -3.56685 & 2.27946 \\
\hline mmu-miR-1939_mat & 0.0208102 & -5.05438 & 3.73398 \\
\hline mmu-miR-1306_mat & 0.012169 & -7.46789 & 2.87234 \\
\hline mmu-miR-742 & 0.00173378 & -12.9825 & 22.0727 \\
\hline mmu-miR-1224_mat & 0.0184421 & -16.0222 & 19.4625 \\
\hline
\end{tabular}

macrophages, monocytes, and other leucocytes. Some also have been associated with $\mathrm{AMD}$, including $\mathrm{C} 3, \mathrm{Ccl} 2$, and Cx3CL1. ${ }^{34-36}$

\section{Expression Time Course of miRNas After Light Exposure}

In this study, we find 8 of 37 miRNAs that are involved in various aspects of regulation of the immune response. Further, our qPCR findings showed that seven of these are modulated across the 7-day time course of the experiment. The MiR- $467 \mathrm{~d}$ showed inconsistent and/or undetectable fold change values across the time course, potentially due to low target abundance. Hence, its significant upregulation in the miRNA array analysis could be attributed to a false-positive result as a result of the "Monte Carlo effect." 37

While all seven of the eight selected miRNAs are upregulated after 24 hours of light damage, in parallel with the TUNEL-positive cells in the ONL, only two (351 and 542$3 \mathrm{p})$ continued to rise beyond 3 days of exposure. Those that reached peak expression at 3 days most likely have roles in the acute phase of retinal damage, while 351 and 542-3p are more likely to mediate changes in the retinal environment during the postacute phase of degeneration in this model. While miR-351 is associated with neuronal and myogenic progenitor cell differentiation, ${ }^{38,39}$ and miR-542-3p has been reported to inhibit tumor angiogenesis, ${ }^{40}$ their roles in retinal remodeling after LD remain to be determined.

The long-term effects of acute bright light exposure are well known ${ }^{13,14}$ and these new data provide insight into genes that may be key players during this phase. The targets of these two miRNAs include the chemokines CXCL1 and 10, IL6, TNF, and CD276. Chemokines CXCL1 and 10 are small molecules belonging to the CXC chemokine family and function as chemoattractants responsible for leukocyte trafficking. While not much is known about their role in AMD or light-induced retinal degeneration, they have been shown to facilitate the recruitment of lymphocytes to lesion site in atherosclerosis and other inflammatory conditions of the cardiovascular system. ${ }^{41}$ Both IL6 and TNF are potent proinflammatory cytokines implicated in a wide variety of inflammationassociated disease states. Of these TNF- $\alpha$ (one of the most common forms of TNF) has been looked at extensively in AMD and shown to express readily by macrophages present in the choroidal neovascular (CNV) membranes of AMD patients. ${ }^{42}$ Additionally, anti-TNF agents are being used currently as a therapeutic strategy for wet AMD. ${ }^{43}$

Five miRNA demonstrate peak expression at 3 days after exposure. Of particular interest is the upregulation of miR-155 (increased 55-fold), which facilitates the inflammatory response $^{44}$ and targets complement factor $\mathrm{H}(\mathrm{CFH}),{ }^{45}$ a major inhibitor of the alternative complement pathway. The $\mathrm{Y} 402 \mathrm{H}$ $\mathrm{SNP}$ in the $C F H$ gene is a major risk factor for AMD and multiple variants confer elevated or reduced risk of the disease. ${ }^{9,11}$ MicroRNA-155 binds directly to the 3'-UTR of $\mathrm{CFH}$ to facilitate activation of the complement pathway in Alzheimer's disease. ${ }^{46}$ In vitro, miR-155 favors the proinflammatory (M1) polarization of immune cells by repressing expression of anti-inflammatory (M2) characteristic proteins, ${ }^{47,48}$ and miR-155-deficient mice have a reduced inflammatory macrophages response. ${ }^{21}$ Peak expression of miR-155 in this model at 3 days after light exposure, correlates well with

TABLE 3. Highly Represented Clusters of Biological Functions Modulated by Candidate miRNAs

\begin{tabular}{|c|c|c|c|}
\hline Biological Process & Enrichment Score & Enrichment $P$ Value & GO ID \\
\hline Positive regulation of cell proliferation & 22.5502 & $1.61 \mathrm{E}-10$ & GO: 8284 \\
\hline Inflammatory response & 19.9534 & 2.16E-09 & GO: 6954 \\
\hline Positive regulation of transcription from RNA polymerase II promoter & 19.7899 & $2.54 \mathrm{E}-09$ & GO: 45944 \\
\hline Angiogenesis & 19.3955 & $3.77 \mathrm{E}-09$ & GO: 1525 \\
\hline Negative regulation of cell proliferation & 17.4925 & 2.53E-08 & GO: 8285 \\
\hline Cell adhesion & 16.9623 & 4.30E-08 & GO: 7155 \\
\hline Cell-cell adhesion & 16.9308 & $4.44 \mathrm{E}-08$ & GO: 16337 \\
\hline Negative regulation of transcription, DNA-dependent & 16.8136 & 4.99E-08 & GO: 45892 \\
\hline Transcription, DNA-dependent & 16.5114 & $6.75 \mathrm{E}-08$ & GO: 6351 \\
\hline Transport & 16.0281 & $1.09 \mathrm{E}-07$ & GO: 6810 \\
\hline
\end{tabular}


TABLE 4. List of miRNAs and the Predicted Gene Targets Implicated in Inflammation

\begin{tabular}{|c|c|c|}
\hline miRNA ID & Gene Symbol & Gene Names \\
\hline mmu-miR-467d & $\mathrm{Il} 20 \mathrm{rb}$ & Interleukin 20 receptor $\beta$ \\
\hline mmu-miR-467c & $C d 14$ & CD14 antigen \\
\hline mmu-miR-466h & Myd88 & Myeloid differentiation factor 88 \\
\hline mmu-miR-466d-5p & Rela & V-rel reticuloendotheliosis viral oncogene homolog A \\
\hline mmu-miR-125b-3p & Ccl4 & Chemokine (C-C motif) ligand 4 \\
\hline mmu-miR-155 & Chst2 & Carbohydrate sulfotransferase 2 \\
\hline mmu-miR-207 & $I l 1 b$ & Interleukin $1, \beta$ \\
\hline mmu-miR-351 & Tnfrsf1a & Tumor necrosis factor receptor superfamily, member 1a \\
\hline mmu-miR-449a & $I l 23 r$ & Interleukin 23 receptor \\
\hline mmu-miR-494 & Cxcl11 & Chemokine (C-X-C motif) ligand 11 \\
\hline mmu-miR-509-3p & Gal & Galanin prepropeptide \\
\hline mmu-miR-542-3p & Ccl12 & Chemokine (C-C motif) ligand 12 \\
\hline mmu-miR-685 & Nfkbiz & Nuclear factor of $\kappa$ light polypeptide gene enhancer in B-cells inhibitor, $\zeta$ \\
\hline rno-miR-347 & $\mathrm{Ccl} 7$ & Chemokine (C-C motif) ligand 7 \\
\hline mmu-miR-300 & Cxcl10 & Chemokine (C-X-C motif) ligand 10 \\
\hline mmu-miR-715 & Il6 & Interleukin 6 \\
\hline mmu-miR-720 & $\operatorname{Tnf}$ & Tumor necrosis factor \\
\hline mmu-miR-220 & $\mathrm{Ccl} 3$ & Chemokine (C-C motif) ligand 3 \\
\hline \multirow[t]{12}{*}{ mmu-miR-582-3p } & Cxcl1 & Chemokine (C-X-C motif) ligand 1 \\
\hline & Spn & Sialophorin \\
\hline & $\operatorname{Hmox} 1$ & Heme oxygenase (decycling) 1 \\
\hline & Agt & Angiotensinogen \\
\hline & $J a k 2$ & Janus kinase 2 \\
\hline & Sbno2 & Strawberry notch homolog 2 \\
\hline & Clcf1 & Cardiotrophin-like cytokine factor 1 \\
\hline & Zfp36 & Zinc finger protein 36 \\
\hline & $C d 276$ & CD276 antigen \\
\hline & Ier3 & Immediate early response 3 \\
\hline & Pla2g $4 a$ & Phospholipase A2, group IVA \\
\hline & Alox 5 ap & Arachidonate 5-lipoxygenase activating protein \\
\hline
\end{tabular}

our previous findings showing peak M1 immune cell recruitment at the site of damage at 3 days. ${ }^{15}$

MicroRNA-207 is upregulated in response to neurotrophins, ${ }^{49}$ that promote photoreceptor cell survival. ${ }^{50}$ Peak expression of mir-207 at 3 days may reflect an attempt by the retina to protect its remaining photoreceptor cell population. In addition, upregulation of miR-207 might sustain the downregulation of one of its predicted targets carbohydrate (N-acetylglucosamine-6-O) sulfotransferase 2 (Chst2), which stimulates the formation of L-selectins on vascular endothelial cell surfaces, to mediate adhesion of lymphocytes around the sites of inflammation. ${ }^{51}$ Less is known about miR-125b-3p, which also had peak expression at 3 days after light exposure, and recently been shown to be a potential biomarker for inflammatory bowel disease. ${ }^{52}$

Two other miRNAs reaching peak expression at $3 \mathrm{~d}$ are miR347 and miR-449a. Neither has been implicated previously in modulating the inflammatory immune response. MicroRNA347 promotes neuronal apoptosis ${ }^{53}$ and miR-449a is a known tumor suppressor that promotes cell death. ${ }^{54,55}$ Our analysis showed that all five miRNAs have putative binding sites in the $3^{\prime}$ UTR s of chemokine, cytokine, and other inflammatory effector proteins (Table 4), indicating that further characterization of their roles in inflammation is warranted.

It is evident from the above expression profiles that the majority of the inflammation related miRNAs show a rapid and sustained increase in expression due to bright light exposure, implying a stricter translational control of their respective targets. However, we know from our previous studies that many cytokines and chemokines (including the ones identified as targets in this study) exhibit increased expression due to bright light exposure. ${ }^{15,23}$ This discrepancy in the expression levels of miRNAs and their target genes could be a facet of the complex regulatory networks, compensatory or otherwise, that are at play inside cells/tissues during ageing, disease, and/ or damage. Furthermore, there is a growing body of evidence supporting the hypothesis that expression levels of some miRNAs may be directly proportional to the amount of target sites available. A phenomenon known as "target mediated miRNA protection" (TMMP). ${ }^{56}$

We also analyzed the expression pattern of miRNAs of the miR183/96/182 cluster (Fig. 4D), which have been shown to occur selectively in the photoreceptor layer. Previous studies have implied the role of this cluster in normal photoreceptor morphogenesis and functioning, maintaining the retinal circadian cycle and having a protective role in bright lightinduced retinal degeneration. ${ }^{57,58}$ Unlike Zhu et al., ${ }^{59}$ who showed an upregulation of these miRNAs due to environmental light conditions (30-minute exposure to 10,000 lux light), our model showed a downward expression trend under a more sustained (24-hour) exposure of bright (1000 lux) light. This discrepancy could be due to the more chronic degenerative state of the retina in our experimental paradigm inherent in retinal degenerations.

\section{Conchusions}

In this study, we identified 37 miRNAs upregulated by LD, including seven that regulated the inflammatory response, which is a key mediator of retinal degeneration in this model, and in AMD. The miRNAs are endogenously-occurring molecules that can be safely introduced in vivo without triggering a nonspecific immune response. Because these miRNAs regulate multiple genes and pathways simultaneously, they provide new 


\section{A: Peak expression 3 days after LD (>10 fold)}

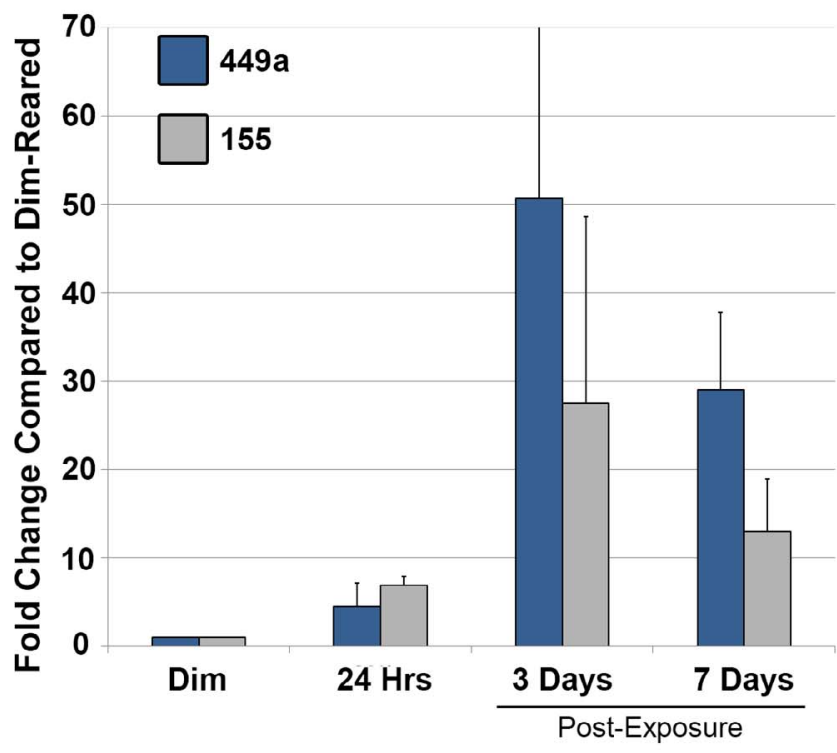

\section{C: Peak expression 7 days after LD}

\section{B: Peak expression 3 days after LD ( $<10$ fold)}

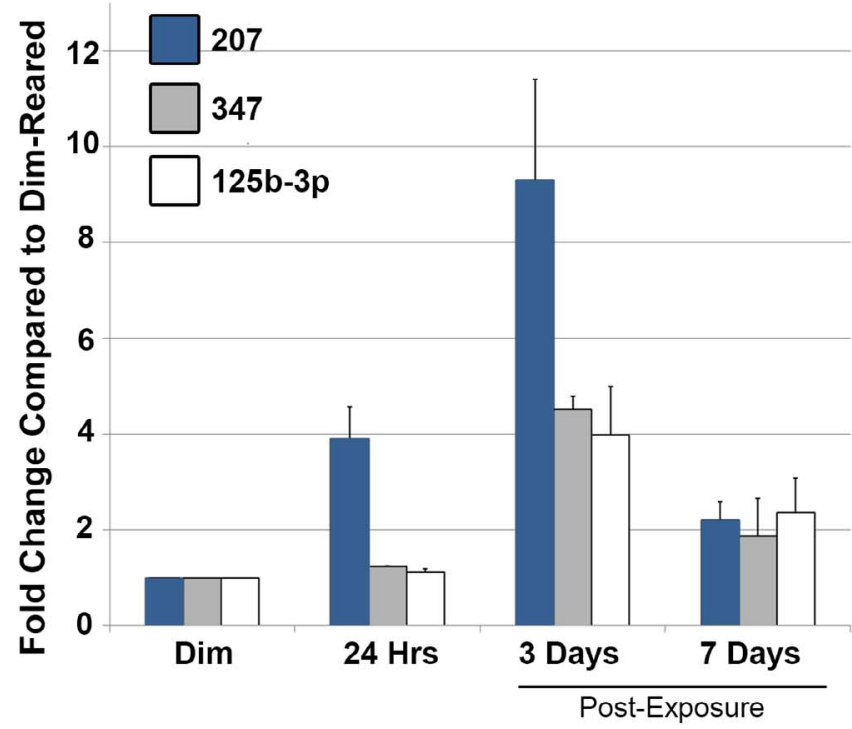

D: Reduced expression across LD timecourse

Figure 4. Expression of 10 miRNAs at 24 hours, and 3 and 7 days after bright light exposure. (A, B) MicroRNAs showing highest levels of expression at 3 days after exposure. (C) MicroRNAs showing progressive upregulation in the postexposure period peaking at 7 days after exposure. (D) MicroRNAs showing downregulation at 24 hours and during the postexposure period. The expression trend for all miRNAs was significant based on a 1-way ANOVA analysis $(P<0.05)$

potential therapeutic targets with far-reaching biological outcomes suitable for management of complex retinal disorders, like AMD.

\section{Acknowledgments}

Supported by the Australian Research Council Centres of Excellence Program Grant CE0561903 and National Health and Medical Research Council Grant 1049990.

Disclosure: K. Saxena, None; M.V. Rutar, None; J.M. Provis, None; R.C. Natoli, None

\section{References}

1. Ambros V, Bartel B, Bartel DP, et al. A uniform system for microRNA annotation. RNA. 2003;9:277-279.

2. Johnson CD, Esquela-Kerscher A, Stefani G, et al. The let-7 microRNA represses cell proliferation pathways in human cells. Cancer Res. 2007;67:7713-7722.

3. Chang TC, Wentzel EA, Kent OA, et al. Transactivation of miR$34 \mathrm{a}$ by $\mathrm{p} 53$ broadly influences gene expression and promotes apoptosis. Mol Cell. 2007;26:745-752. 
4. O'Connell RM, Rao DS, Baltimore D. MicroRNA regulation of inflammatory responses. Annu Rev Immunol. 2012;30:295312.

5. Kosik KS. The neuronal microRNA system. Nat Rev Neurosci. 2006;7:911-920.

6. Rao P, Benito E, Fischer A. MicroRNAs as biomarkers for CNS disease. Front Mol Neurosci. 2013;6:39.

7. Pogue AI, Hill JM, Lukiw WJ. MicroRNA (miRNA): sequence and stability, viroid-like properties, and disease association in the CNS. Brain Res. 2014;1584:73-79.

8. Penfold PL, Killingsworth MC, Sarks SH. Senile macular degeneration. The involvement of giant cells in atrophy of the retinal pigment epithelium. Invest Ophthalmol Vis Sci. 1986;27:364-371.

9. Hageman GS, Anderson DH, Johnson LV, et al. A common haplotype in the complement regulatory gene factor $\mathrm{H}$ (HF1/ $\mathrm{CFH})$ predisposes individuals to age-related macular degeneration. Proc Natl Acad Sci U S A. 2005;102:7227-7232.

10. Klein RJ, Zeiss C, Chew EY, et al. Complement factor $\mathrm{H}$ polymorphism in age-related macular degeneration. Science. 2005;308:385-389.

11. Haines JL, Hauser MA, Schmidt S, et al. Complement factor $\mathrm{H}$ variant increases the risk of age-related macular degeneration. Science. 2005;308:419-421.

12. Fritsche LG, Fariss RN, Stambolian D, Abecasis GR, Curcio CA, Swaroop A. Age-related macular degeneration: genetics and biology coming together. Annu Rev Genomics Hum Genet. 2014; 15:151-171.

13. Marc RE, Jones BW, Watt CB, Vazquez-Chona F, Vaughan DK, Organisciak DT. Extreme retinal remodeling triggered by light damage: implications for age related macular degeneration. Mol Vis. 2008;14:782-806.

14. Rutar M, Provis JM, Valter K. Brief exposure to damaging light causes focal recruitment of macrophages, and long-term destabilization of photoreceptors in the albino rat retina. Curr Eye Res. 2010;35:631-643.

15. Rutar M, Natoli R, Valter K, Provis JM. Early focal expression of the chemokine $\mathrm{Ccl} 2$ by Muller cells during exposure to damage-inducing bright continuous light. Invest Ophthalmol Vis Sci. 2011;52:2379-2388.

16. Rutar M, Natoli R, Kozulin P, Valter K, Gatenby P, Provis JM. Analysis of complement expression in light-induced retinal degeneration: synthesis and deposition of C3 by microglia/ macrophages is associated with focal photoreceptor degeneration. Invest Ophthalmol Vis Sci. 2011;52:5347-5358.

17. Rutar M, Natoli R, Provis JM. Small interfering RNA-mediated suppression of $\mathrm{Ccl} 2$ in Muller cells attenuates microglial recruitment and photoreceptor death following retinal degeneration. J Neuroinflammation. 2012;9:221.

18. Rutar M, Valter K, Natoli R, Provis JM. Synthesis and propagation of complement C3 by microglia/monocytes in the aging retina. PLoS One. 2014;9:e93343.

19. Sonkoly E, Stahle M, Pivarcsi A. MicroRNAs and immunity: novel players in the regulation of normal immune function and inflammation. Semin Cancer Biol. 2008;18:131-140.

20. O'Neill LA, Sheedy FJ, McCoy CE. MicroRNAs: the fine-tuners of Toll-like receptor signalling. Nat Rev Immunol. 2011;11: 163-175.

21. Du F, Yu F, Wang Y, et al. MicroRNA-155 deficiency results in decreased macrophage inflammation and attenuated atherogenesis in apolipoprotein e-deficient mice. Arterioscler Thromb Vasc Biol. 2014;34:759-767.

22. Taganov KD, Boldin MP, Chang KJ, Baltimore D. NF-kappaBdependent induction of microRNA miR-146, an inhibitor targeted to signaling proteins of innate immune responses. Proc Natl Acad Sci US A. 2006;103:12481-12486.
23. Natoli R, Zhu Y, Valter K, Bisti S, Eells J, Stone J. Gene and noncoding RNA regulation underlying photoreceptor protection: microarray study of dietary antioxidant saffron and photobiomodulation in rat retina. Mol Vis. 2010;16:18011822.

24. Noell WK, Walker VS, Kang BS, Berman S. Retinal damage by light in rats. Invest Ophthalmol. 1966;5:450-473.

25. Battelle BA, LaVail MM. Rhodopsin content and rod outer segment length in albino rat eyes: modification by dark adaptation. Exp Eye Res. 1978;26:487-497.

26. Ortin-Martinez A, Jimenez-Lopez M, Nadal-Nicolas FM, et al. Automated quantification and topographical distribution of the whole population of S- and L-cones in adult albino and pigmented rats. Invest Ophthalmol Vis Sci. 2010;51:31713183.

27. Marco-Gomariz MA, Hurtado-Montalban N, Vidal-Sanz M, Lund RD, Villegas-Perez MP. Phototoxic-induced photoreceptor degeneration causes retinal ganglion cell degeneration in pigmented rats. J Comp Neurol. 2006;498:163-179.

28. Langmann T. Microglia activation in retinal degeneration. $J$ Leukoc Biol. 2007;81:1345-1351.

29. Tezel G, Yang X, Luo C, Peng Y, Sun SL, Sun D. Mechanisms of immune system activation in glaucoma: oxidative stressstimulated antigen presentation by the retina and optic nerve head glia. Invest Ophthalmol Vis Sci. 2007;48:705-714.

30. Bringmann A, Iandiev I, Pannicke T, et al. Cellular signaling and factors involved in Muller cell gliosis: neuroprotective and detrimental effects. Prog Retin Eye Res. 2009;28:423-451.

31. Rohrer B, Guo Y, Kunchithapautham K, Gilkeson GS. Eliminating complement factor D reduces photoreceptor susceptibility to light-induced damage. Invest Ophthalmol Vis Sci. 2007;48:5282-5289.

32. Zhang C, Shen JK, Lam TT, et al. Activation of microglia and chemokines in light-induced retinal degeneration. Mol Vis. 2005;11:887-895.

33. Chen L, Wu W, Dentchev T, et al. Light damage induced changes in mouse retinal gene expression. Exp Eye Res. 2004; 79:239-247.

34. Ambati J, Anand A, Fernandez S, et al. An animal model of agerelated macular degeneration in senescent Ccl-2- or Ccr-2deficient mice. Nat Med. 2003;9:1390-1397.

35. Jonas JB, Tao Y, Neumaier M, Findeisen P. Monocyte chemoattractant protein 1 , intercellular adhesion molecule 1 , and vascular cell adhesion molecule 1 in exudative age-related macular degeneration. Arch Ophthalmol. 2010;128:12811286.

36. Raoul W, Auvynet C, Camelo S, et al. CCL2/CCR2 and CX3CL1/ CX3CR1 chemokine axes and their possible involvement in age-related macular degeneration. J Neuroinflammation. 2010;7:87.

37. Bustin SA, Nolan T. Pitfalls of quantitative real-time reversetranscription polymerase chain reaction. J Biomol Tech. 2004; 15:155-166.

38. Li X, Feng R, Huang C, et al. MicroRNA-351 regulates TMEM 59 (DCF1) expression and mediates neural stem cell morphogenesis. RNA Biol. 2012;9:292-301.

39. Chen Y, Melton DW, Gelfond JA, McManus LM, Shireman PK. MiR-351 transiently increases during muscle regeneration and promotes progenitor cell proliferation and survival upon differentiation. Physiol Genomics. 2012;44:1042-1051.

40. He T, Qi F, Jia L, et al. MicroRNA-542-3p inhibits tumour angiogenesis by targeting angiopoietin-2. J Pathol. 2014;232: 499-508.

41. van den Borne P, Quax PH, Hoefer IE, Pasterkamp G. The multifaceted functions of CXCL10 in cardiovascular disease. Biomed Res Int. 2014;2014:893106. 
42. Oh $\mathrm{H}$, Takagi $\mathrm{H}$, Takagi $\mathrm{C}$, et al. The potential angiogenic role of macrophages in the formation of choroidal neovascular membranes. Invest Ophthalmol Vis Sci. 1999;40:1891-1898.

43. Theodossiadis PG, Liarakos VS, Sfikakis PP, Vergados IA, Theodossiadis GP. Intravitreal administration of the anti-tumor necrosis factor agent infliximab for neovascular age-related macular degeneration. Am J Ophthalmol. 2009;147:825-830.

44. Kutty RK, Nagineni CN, Samuel W, Vijayasarathy C, Hooks JJ, Redmond TM. Inflammatory cytokines regulate microRNA-155 expression in human retinal pigment epithelial cells by activating JAK/STAT pathway. Biochem Biophys Res Commun. 2010;402:390-395.

45. Lukiw WJ, Surjyadipta B, Dua P, Alexandrov PN. Common micro RNAs (miRNAs) target complement factor $\mathrm{H}$ (CFH) regulation in Alzheimer's disease $(\mathrm{AD})$ and in age-related macular degeneration (AMD). Int J Biochem Mol Biol. 2012;3: 105-116.

46. Lukiw WJ, Alexandrov PN. Regulation of complement factor $\mathrm{H}$ (CFH) by multiple miRNAs in Alzheimer's disease (AD) brain. Mol Neurobiol. 2012;46:11-19.

47. Ponomarev ED, Veremeyko T, Weiner HL. MicroRNAs are universal regulators of differentiation, activation, and polarization of microglia and macrophages in normal and diseased CNS. Glia. 2013;61:91-103.

48. Elton TS, Selemon H, Elton SM, Parinandi NL. Regulation of the MIR155 host gene in physiological and pathological processes. Gene. 2013;532:1-12.

49. Montalban E, Mattugini N, Ciarapica R, et al. MiR-21 is an Ngfmodulated microRNA that supports Ngf signaling and regulates neuronal degeneration in PC12 cells. Neuromolecular Med. 2014;16:415-430.

50. LaVail MM, Unoki K, Yasumura D, Matthes MT, Yancopoulos GD, Steinberg RH. Multiple growth factors, cytokines, and neurotrophins rescue photoreceptors from the damaging effects of constant light. Proc Natl Acad Sci U S A. 1992;89: 11249-11253.

51. Li X, Tu L, Murphy PG, Kadono T, Steeber DA, Tedder TF. CHST1 and CHST2 sulfotransferase expression by vascular endothelial cells regulates shear-resistant leukocyte rolling via L-selectin. J Leukoc Biol. 2001;69:565-574.

52. Coskun M, Bjerrum JT, Seidelin JB, Troelsen JT, Olsen J. Nielsen OH. miR-20b, miR-98, miR-125b-1*, and let-7e* as new potential diagnostic biomarkers in ulcerative colitis. World $J$ Gastroenterol. 2013;19:4289-4299.

53. Gubern C, Camos S, Ballesteros I, et al. miRNA expression is modulated over time after focal ischaemia: up-regulation of miR-347 promotes neuronal apoptosis. FEBS J. 2013;280: 6233-6246.

54. Ren XS, Yin MH, Zhang X, et al. Tumor-suppressive microRNA449 a induces growth arrest and senescence by targeting E2F3 in human lung cancer cells. Cancer Lett. 2014;344:195-203.

55. Wei B, Song Y, Zhang Y, Hu M. microRNA-449a functions as a tumor-suppressor in gastric adenocarcinoma by targeting Bcl2. Oncol Lett. 2013;6:1713-1718.

56. Chatterjee S, Fasler M, Bussing I, Grosshans H. Target-mediated protection of endogenous microRNAs in C. elegans. Dev Cell. 2011;20:388-396.

57. Lumayag S, Haldin CE, Corbett NJ, et al. Inactivation of the microRNA-183/96/182 cluster results in syndromic retinal degeneration. Proc Natl Acad Sci U S A. 2013;110:E507-E516.

58. Krol J, Busskamp V, Markiewicz I, et al. Characterizing lightregulated retinal microRNAs reveals rapid turnover as a common property of neuronal microRNAs. Cell. 2010;141: 618-631.

59. Zhu Q, Sun W, Okano K, et al. Sponge transgenic mouse model reveals important roles for the microRNA-183 (miR-183)/96/ 182 cluster in postmitotic photoreceptors of the retina. J Biol Chem. 2011;286:31749-31760. 